\title{
Emerging Methods to Objectively Assess Pruritus in Atopic Dermatitis
}

\author{
Mary Patricia Smith · Karen Ly - Quinn Thibodeaux - Thulasi Weerasinghe • \\ Jashin J. Wu • Gil Yosipovitch • Tina Bhutani • Wilson Liao
}

Received: May 31, 2019 / Published online: June 29, 2019

(C) The Author(s) 2019

\begin{abstract}
Introduction: Atopic dermatitis (AD) is an inflammatory skin disease with a chronic, relapsing course. Clinical features of AD vary by age, duration, and severity but can include papules, vesicles, erythema, exudate, xerosis, scaling, and lichenification. However, the most defining and universal symptom of $\mathrm{AD}$ is pruritus. Pruritus or itch, defined as an unpleasant urge to scratch, is problematic for many reasons, particularly its negative impact on quality of life. Despite the profoundly negative impact of pruritus on patients with $\mathrm{AD}$, clinicians and researchers lack standardized and validated
\end{abstract}

Enhanced Digital Features To view enhanced digital features for this article go to https://doi.org/10.6084/ m9.figshare.8320133.

M. P. Smith $(\varangle) \cdot$ K. Ly · Q. Thibodeaux ·

T. Weerasinghe $\cdot$ T. Bhutani $\cdot$ W. Liao

Department of Dermatology, University of

California, San Francisco, CA, USA

e-mail: mary.smith2@ucsf.edu

\section{J. J. Wu}

Dermatology Research and Education Foundation, Irvine, CA, USA

G. Yosipovitch

Department of Dermatology and Cutaneous Surgery, University of Miami Miller School of Medicine, Miami, FL, USA methods to objectively measure pruritus. The purpose of this review is to discuss emerging methods to assess pruritus in AD by describing objective patient-centered tools developed or enhanced over the last decade that can be utilized by clinicians and researchers alike.

Methods: This review is based on a literature search in Medline, Embase, and Web of Science databases. The search was performed in February 2019. The keywords were used "pruritus," "itch," "atopic dermatitis," "eczema," "measurements," "tools," "instruments," "accelerometer," "wrist actigraphy," "smartwatch," "transducer," "vibration," "brain mapping," "magnetic resonance imaging," and "positron emission tomography." Only articles written in English were included, and no restrictions were set on study type. To focus on emerging methods, prioritization was given to results from the last decade (2009-2019).

Results: The search yielded 49 results in PubMed, 134 results in Embase, and 85 results in Web of Science. Each result was independently reviewed in a standardized manner by two of the authors (M.S., K.L.), and disagreements between reviewers were resolved by consensus. Relevant findings were categorized into the following sections: video surveillance, acoustic surveillance, wrist actigraphy, smart devices, vibration transducers, and neurological imaging. Examples are provided along with descriptions of how each technology works, instances of use in research or clinical practice, 
and as applicable, reports of validation studies and correlation with other methods.

Conclusion: The variety of new and improved methods to evaluate pruritus in $\mathrm{AD}$ is welcomed by clinicians, researchers, and patients alike. Future directions include next-generation smart devices as well as exploring new territories, such as identifying biomarkers that correlate to itch and machine-learning programs to identify itch processing in the brain. As these efforts continue, it will be essential to remain patientcentered by developing techniques that minimize discomfort, respect privacy, and provide accurate data that can be used to better manage itch in AD.

Keywords: Atopic dermatitis; Diagnosis; Eczema; Itch; Pruritus

\section{INTRODUCTION}

Atopic dermatitis (AD) is an inflammatory skin disease with a chronic, relapsing course. Clinical features of AD vary by age, duration, and severity but can include papules, vesicles, erythema, exudate, xerosis, scaling, and lichenification. However, the most defining and universal symptom of $\mathrm{AD}$ is pruritus. Pruritus or itch, defined as an unpleasant urge to scratch, is problematic for many reasons, particularly its negative impact on quality of life. In fact, pruritus in $\mathrm{AD}$ can be so severe and unrelenting that it contributes to mental health consequences ranging from hyperactivity $[1,2]$ to generalized anxiety and even major depressive disorders [3-5] in some patients.

Despite the profoundly negative impact of pruritus on patients with $\mathrm{AD}$, clinicians and researchers lack standardized and validated methods to objectively measure pruritus [6]. Since pruritus is an internalized sensation, assessment typically hinges on subjective patient reporting. A variety of scales and questionnaires have been developed for this purpose. One of the most commonly used is the Peak Pruritus Numerical Rating Scale (NRS), which consists of a single question for the patient: "On a scale of $0-10$, with 0 being 'no itch' and 10 being 'worst itch imaginable,' how would you rate your itch at the worst moment during the previous $24 \mathrm{~h}$ ?" The Peak Pruritus NRS was recently reported to be a well-defined, reliable, sensitive, and valid scale for evaluating worst itch intensity [7], and its efficient and simple format makes it particularly attractive to busy clinicians.

While such tools can be very useful in clinical practice, patient-reported assessments are less useful for research since biases arise from differences in how an individual experiences and describes pruritus. A " 10 out of 10 " or "worst itch imaginable" for one person might be sensed much less severely by another person. For example, a patient with years of chronic itch may experience neural sensitization or desensitization that can skew their self-reporting [8-13]. Similarly, "itchy" is a common way to describe pruritus, but some individuals may experience it as a "burning" or a "tingling" sensation, potentially resulting in their pruritus being inadequately captured by certain clinical tools.

As these examples show, scales and questionnaires might be helpful for a clinician treating an individual patient with $\mathrm{AD}$, but complementary and more objective tools are needed to rigorously research this condition, such as detecting subtle variations and comparing cohorts before and after an $\mathrm{AD}$ treatment or intervention $[14,15]$. The purpose of this review is to discuss emerging methods to assess pruritus in $\mathrm{AD}$ by describing objective patientcentered tools developed or enhanced over the last decade that can be utilized by clinicians and researchers alike. Examples are provided along with descriptions of how each technology works, instances of use in research or clinical practice, and as applicable, reports of validation studies and correlation with other methods to measure pruritus. Several of these methods measure pruritus indirectly by quantifying scratch, but several new technologies provide novel ways to measure pruritus directly and objectively. Given the centrality of pruritus in $\mathrm{AD}$, there is a need for more tools that allow clinicians and researchers to accurately measure this symptom in individual patients as well as cohorts with AD. 


\section{METHODS}

This review is based on a literature search in Medline, Embase, and Web of Science databases. The search was performed in February 2019. The following primary keywords were used: "pruritus," "itch," "atopic dermatitis," "eczema," "measurements," "tools," and "instruments." Preliminary results were reviewed, and additional searches were conducted by linking these primary keywords with "AND" and "OR" statements to the following secondary keywords: "accelerometer," "wrist actigraphy," "smartwatch," "transducer," "vibration," "brain mapping," "magnetic resonance imaging," and "positron emission tomography." These secondary keywords were selected to narrow results to objective methods rather than subjective instruments like scales or questionnaires. When applicable, corresponding Medical Subject Headings (MeSH) were used. Only articles written in English were included, and no restrictions were set on study type. To focus on emerging methods, prioritization was given to results from the last decade (2009-2019). This review is based on previously conducted studies and does not contain any studies with human participants or animals performed by any of the authors.

\section{RESULTS}

The search yielded 49 results in PubMed, 134 results in Embase, and 85 results in Web of Science. Each result was independently reviewed in a standardized manner by two of the authors (M.S., K.L.), and disagreements between reviewers were resolved by consensus. Relevant findings were categorized into the sections that follow, and a summary of these results is presented in Table 1.

\section{Video Surveillance}

Video surveillance is not a new method to assess pruritus in $\mathrm{AD}$, but it is essential to include in this review for two reasons. First, direct observation is considered the gold standard to measure scratching as a proxy for itch. This involves a trainer evaluator watching patients while they sleep and recording the frequency and duration scratching activity in real time $[16,17]$. Second, technological advances have made it possible to observe scratching via video surveillance by providing recorded footage that can be reviewed at any time and by multiple evaluators.

In recent years, several advancements have further improved this method of pruritus assessment, making it more efficient and accurate. Video surveillance is almost exclusively used to measure nocturnal itch, when the subject has limited activity. A potential challenge of observing scratching behaviors during sleep is that people are generally under covers that can obscure the camera's view. To address this, infrared video cameras have been employed to capture movements without disturbing sleep [18]. Furthermore, researchers have developed standardized criteria to evaluate recordings. For example, "scratching bouts" are typically defined as "an apparent action of rubbing or scratching to any part of the body with a rhythmical movement using hands, fingers, or feet lasting longer than $5 \mathrm{~s}$ " [19]. By applying this criteria, recordings can be analyzed by multiple people, allowing for a comparison of any difference, and ultimately a determination of a quantitative value for itch. This method is useful in that it offers an objective index of overall scratch activity while simultaneously tracking scratching at specific times and locations [18].

Despite the advantages of video surveillance, it is a time-consuming endeavor, and patients often decline this method because of privacy concerns [19]. Furthermore, it is possible that the placement of the recording device and/or the patient's body could result in "blind spots" where scratching behavior is hidden and therefore not counted (e.g., a person sleeping prone with a scratching hand wedged between their abdomen and the mattress). Another limitation is that patients' awareness of being recorded may alter their normal sleeping behavior, such as reducing the depth or duration of sleep or other conscious or unconscious modifications due to the observation. To 


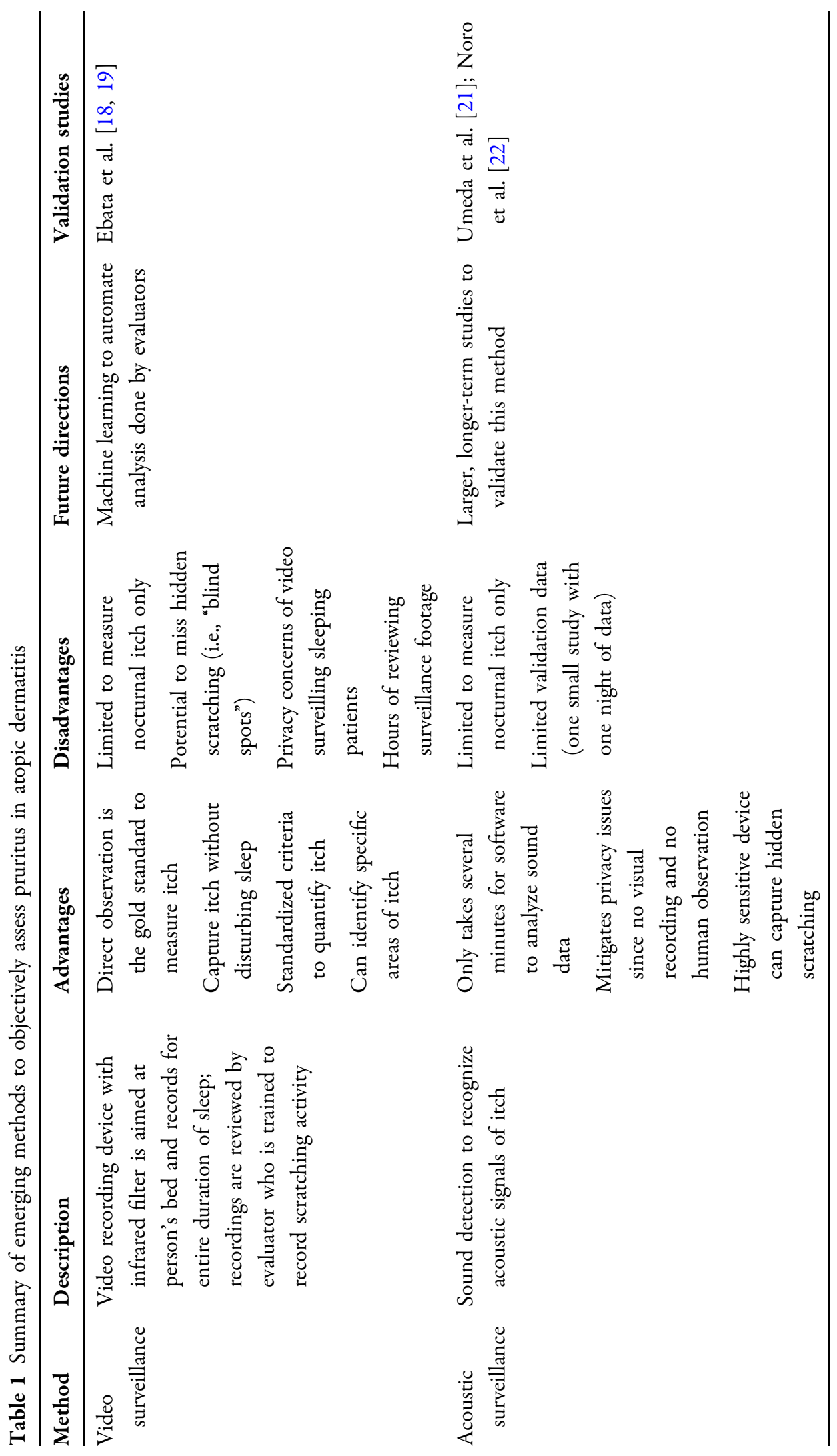




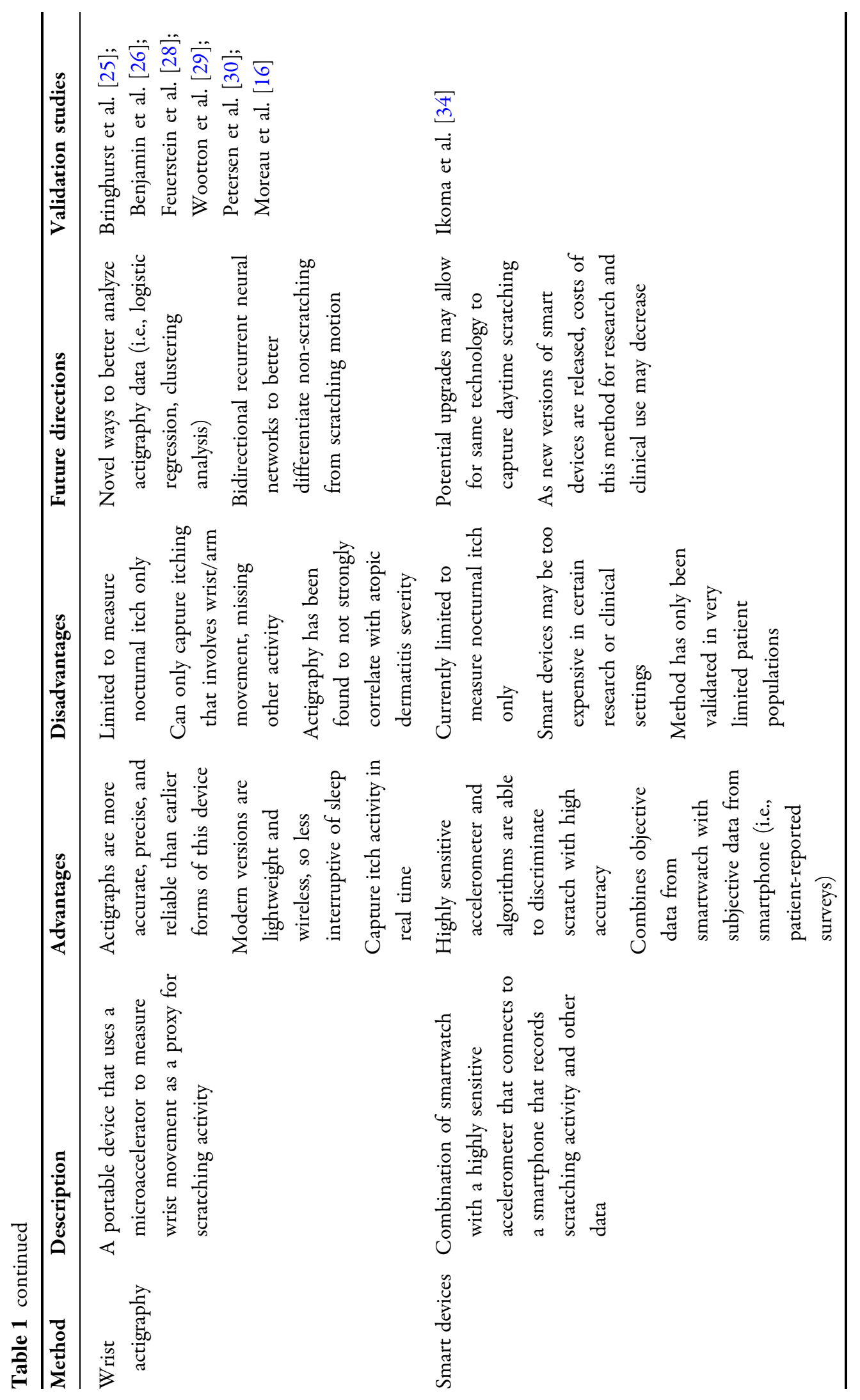




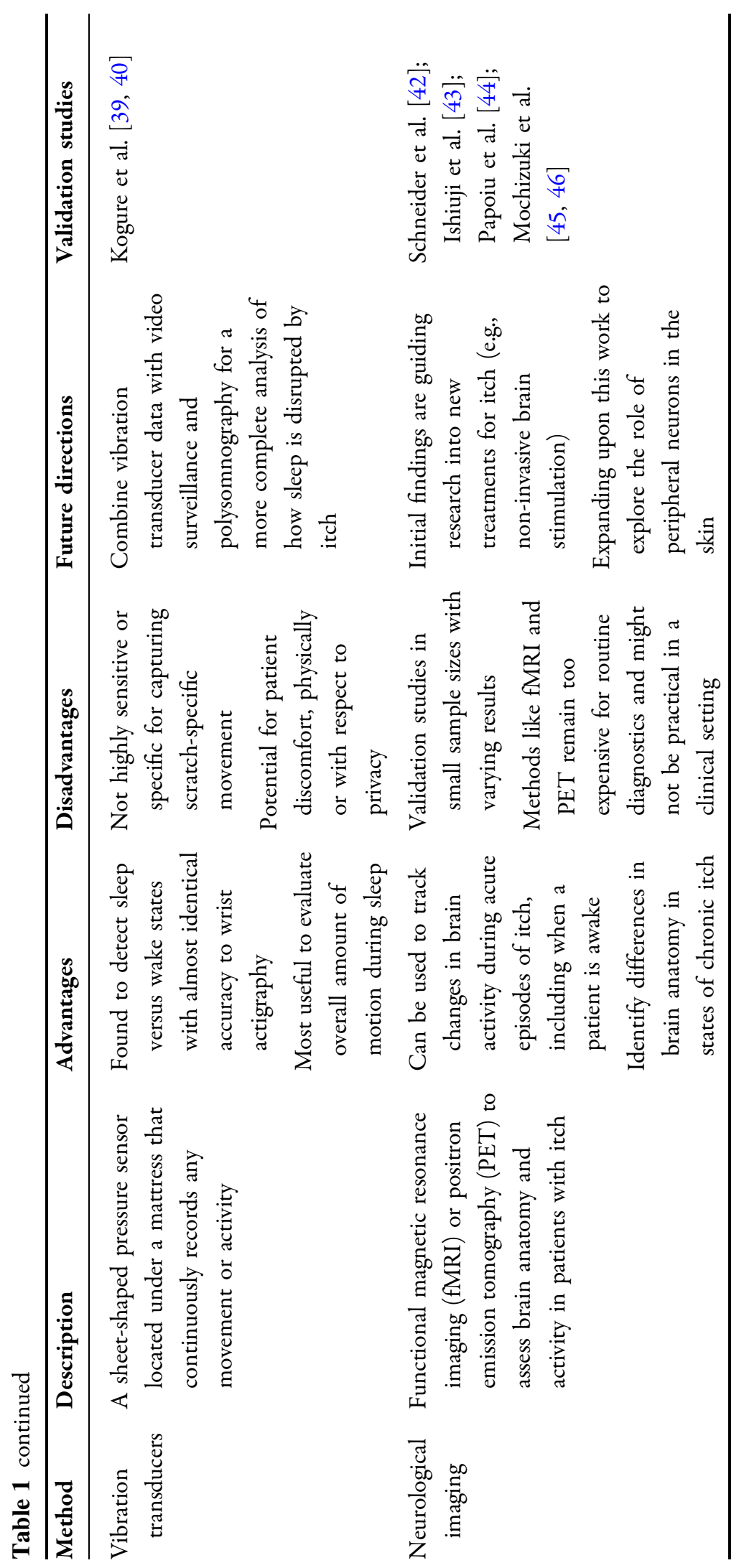


mitigate these issues, new tools are under development that offer less intrusive but more comprehensive and automated methods of visual scratching observation. One key area of development is combining infrared video recordings with machine-learning technologies that can be programmed to "learn" scratching movements [20], including unique movements of an individual person. In this way, a machine can take the place of a human observer, offering the benefits of decreased variations in evaluator judgement or visual fatigue, providing more objective and consistent measurements across patients. Such devices could offer a type of personalized medicine for patients with $\mathrm{AD}$ by evaluating their pruritus at baseline and in response to different interventions.

\section{Acoustic Surveillance}

Given the many disadvantages of video surveillance for measuring pruritus in $\mathrm{AD}$, a similar but alternative method has emerged: acoustic surveillance. This method was first tested in an AD mouse model (interleukin-18 transgenic mice) whereby scratching behavior of these mice was captured using a sound recording device [21]. The scratching sounds, including frequency and wavelength information, were analyzed to develop a software program that could recognize and quantify the mice's scratching behavior, similar to video surveillance but with no direct visualization and with automated and thus much faster rates of scratch data analysis. In this mouse model, results from the acoustic counting system were compared to video surveillance and found to be identical [21].

Building upon this promising mouse model, Noro et al. developed a sound detector with similar acoustic surveillance technology but designed to be worn on the wrist of humans and capture their scratching behavior [22]. Very interestingly, this device did not rely on airconducted noises but rather detected the specific bone-conducted sound made by scratching movements through the finger and wrist bones [22]. To test this novel device, sound data were gathered from healthy controls and patients with $\mathrm{AD}$ while both groups slept over one 6-h period. The subjects' scratching behavior was simultaneously observed by infrared video. The software took a few minutes to analyze the acoustic data compared to several hours of a human observer scoring video recordings. Ultimately, the authors reported that the scratching time captured by the sound detector was nearly identical to results from video surveillance, the gold standard comparison [22]. Thus, the sound detector eliminated the intrusive need for visualization of human behavior while also rapidly increasing the speed and objectivity of analysis. As with video surveillance, efforts are underway to optimize acoustic surveillance with machine-learning algorithms that analyze sound signals to recognize movement and quantify scratching activity [23].

While acoustic recording does offer many advantages over video surveillance, this device has only been tested in one small, brief trial as described above. Additional larger, longer-term studies are needed to fully validate this method in a wider variety of patient populations.

\section{Wrist Actigraphy}

A wrist actigraph is a portable device that uses a microaccelerator to measure nocturnal wrist movement as a proxy for scratching activity that is correlated to itch in patients with $\mathrm{AD}$ [17]. Movement data is collected in two to three planes, stored locally in the device, and seamlessly uploaded for analysis. Although wrist actigraphy has been used for decades [24, 25] recent advancements have improved this technology to be more accurate, precise, and reliable for clinical and research use. Devices are now much smaller and less invasive (e.g., lightweight and wireless), allowing them to be easily used at home with minimal discomfort for the user. In addition, wrist actigraphs are less expensive than before, making this a more affordable options for clinicians and researchers. Furthermore, wrist actigraphy is increasingly being coupled to new methods of analysis to improve usability and accuracy. 
Several studies have reported a strong correlation between wrist actigraphy findings and AD disease activity. Studies by Ebata et al. [24] and Benjamin et al. [26] found a high correlation between pruritus scoring from wrist actigraphs and infrared video recordings. However, both studies noted that although wrist actigraphs are sensitive, increased specificity is needed to distinguish nocturnal scratching from other nocturnal movements. A study by Hon et al. [27] evaluated children with AD and determined that wrist actigraphy strongly correlated with disease severity as captured by SCORing Atopic Dermatitis (SCORAD), an instrument that combines objective components of $\mathrm{AD}$ (i.e., clinician-assessed signs of pruritus, such as excoriation) with subjective symptoms (i.e., patient-reported itch severity using a visual analogue scale). In this study, wrist activities were correlated with both objective SCORAD components and overall SCORAD values among 24 pediatric patients [27]. Such results support the use of wrist actigraphy as an objective approach to measure pruritus in adult and pediatric $\mathrm{AD}$ patient populations.

Despite these advantages, there are several limitations on the use of wrist actigraphy to measure itch. First, wrist actigraphy can only be used to measure nocturnal pruritus, since wrist movement during the day could be due to any number of activities that would be difficult to discriminate from AD-related scratching [28]. Second, actigraphy generally captures itching that involves wrist or arm movement, but depending on placement of the device, it might not record itching with the palm, feet, or slight movements of individual fingers [16]. Furthermore, many patients with $\mathrm{AD}$ have developed scratching techniques that involve rubbing their torso or extremities against each other or a part of their bed, which is unlikely to be detected by a device on the wrist. Third, wrist actigraphs are typically placed on the dominant hand only, so any scratching activity from the non-dominant hand would be missed. Fourth, wrists are a very common site for AD involvement, which is unfortunate since wearing an actigraph could potentially cause further irritation to an area of the body already primed for inflammation. A fifth and largely technical point, several studies have validated the use of wrist actigraphy to measure itch in $\mathrm{AD}$, but other results have challenged it. Most notably, a large, multicenter randomized controlled trial among children with AD failed to find a correlation between wrist actigraphy and disease severity or quality of life [29]. This study also found that wrist actigraphy was not responsive to change, an assessment of how an instrument captures changes in disease status, which would limit its utility in randomized controlled trials and other research [29].

Fortunately, new techniques are being developed to minimize these limitations and optimize wrist actigraphy data for pruritus assessment. Feuerstein et al. evaluated wrist actigraphy data with a clustering analysis designed to differentiate scratching from walking and restless sleep [28]. By including features such as peak frequency and force of movements, the cluster analysis exhibited a high specificity and sensitivity for differentiating walking from restless sleep, demonstrating its ability to distinguish true scratching from other activities [28]. These results were extended by Petersen et al. who incorporated movement features as independent variables in a logistic regression (LR) [30]. This model similarly demonstrated high discriminative ability to separate scratch from other routine movements [30]. Building on this work, Moreau et al. described a two-step approach using high-resolution, three-dimensional wrist actigraphy data: (1) segmenting the data into "no motion," "single handed motion," and "both handed motion," and then (2) discriminating motion segments into scratching and other motion using a bidirectional recurrent neural network (RNN), a type of artificial intelligence that can model dynamic, temporal behavior such as scratching [16]. Results from this approach were compared to infrared video scoring of pruritus among 24 subjects (18 patients with $\mathrm{AD}$ and six healthy controls) and demonstrated similar results [16]. The authors also compared their results to the LR by Petersen et al. described above and concluded that the RNN algorithm provided a better estimation of the total amount of scratching and, perhaps more 
importantly, accurately predicted when scratching occurred [16, 30]. Moreau et al. noted that such capability could be helpful in assessing the effectiveness of a treatment over the course of a night, which is useful data for clinicians managing itch as well as researchers studying medication dosing [16].

Overall, improvements in the devices themselves and the methods used to analyze the generated data have allowed wrist actigraphy to re-emerge and continuously be improved as a method to assess pruritus in AD. Nevertheless, such methods still must be tested in larger patient populations and directly compared to other tools in large and diverse patient populations in order to be fully validated and suitable for clinical or research use.

\section{Smart Devices}

In the last decade, the medical community has leveraged the convenience and computing power of smart devices to learn more about various medical conditions and also deliver novel interventions, such as dietary intake tracking and medication adherence reminders [31, 32]. Pruritus researchers have joined this technological revolution by leveraging the fundamental principles of wrist actigraphy but modernizing it into a smartwatch. In 2015, Lee et al. reported a pilot study in which three subjects wore a smartwatch with an accelerometer to detect scratching. The smartwatch was able to identify scratching behaviors with 98.5-99.0\% accuracy for right-hand scratching motion and 93.3-97.6\% accuracy for left-hand scratching when compared to infrared video surveillance [33].

In 2017, a product named Itch Tracker was developed through a partnership between dermatologists, Nestle Skin Health, and Apple Inc. Recognizing the need to have better objective methods to evaluate itch, this team developed a software application (app) that can be installed to broadly used smartwatches and used to measure nocturnal scratching. The app incorporates an algorithm that analyzes acceleration data from the smartwatch and differentiates scratching from other types of movement on the basis of characteristic wrist motion [34]. Furthermore, the app also has a smartphone interface where the user can answer itch-related questionnaires, allowing for subjective, patientreported data to be combined with the objective, smartwatch-detected data.

Itch Tracker was validated in two proof-ofconcept studies, which included a total of 25 patients with $\mathrm{AD}$ and 10 healthy controls who were simultaneously evaluated with the app and nocturnal video surveillance. In the first study, the investigators found that there was a statistically significant positive correlation between smartwatch-detected and video-captured scratching time per hour, and the second study demonstrated that total scratching duration in $\mathrm{AD}$ patients was significantly longer than in healthy controls and correlated positively with Eczema Area and Severity Index (EASI) scores [35]. The team concluded that the Itch Tracker had high validity and reliability in measuring scratching in AD and conducted a third, larger study with 201 people with similar findings [34]. On the basis of these data, Itch Tracker represents a smart device that can be used to measure scratching and indirectly, but objectively, evaluate the intensity of pruritus among patients with $\mathrm{AD}$. Owing to the simple interface and unobtrusive design of Itch Tracker, it is suitable for widespread use, but further research will be required to validate the app in more diverse patient populations and clinical settings [34].

\section{Vibration Transducers}

Measurement of vibration is another method that is not entirely new but has been updated by modern technologies. In fact, one of the earliest devices to measure itch was called a pruritometer [36]. It consisted of a resistive sensor and amplifier glued to the middle finger of the dominant hand. Fingernail vibration from scratching would induce an electrical signal transmitted to a wristwatch, providing data on the frequency and intensity of scratching $[37,38]$. These devices had several limitations, most notably the discomfort of placement in such a conspicuous site and the high likelihood 
of the device being knocked askew or falling off entirely, thus compromising any data collection.

In recent years, less invasive and more accurate ways to measure itch-related vibration have been developed. In 2017, Kogure and Ebata described the use of a sheet-shaped body vibrometer (SBV) to measure sleep activity among patients with AD [39]. The SBV consists of a sensitive pressure sensor located under a mattress that continuously records any movement or activity [39]. These authors had previously reported the SBV detecting sleep versus wake states with almost identical accuracy to wrist actigraphy in a healthy population [40]. Expanding on this work, the authors compared the SBV to wrist actigraphy in 20 patients with $\mathrm{AD}$ and found the SBV to be most useful for overall sleep evaluation in these patients (i.e., amount of motion during sleep) while wrist actigraphy is better suited to specifically evaluate scratching [39]. Therefore, the SBV may not be the best option for measuring pruritus but it is nonetheless another tool in the kit, especially for evaluating the impact of $\mathrm{AD}$ on sleep architecture among patients with severe itch.

\section{Neurological Imaging}

Neurological imaging has emerged as a particularly novel method to objectively detect functional and anatomical changes in both acute and chronic pruritus. For acute itch, functional magnetic resonance imaging (fMRI) or positron emission tomography (PET) has been used to assess brain activity during experimentally induced itching episodes [41]. In one study, acute histamine-induced itch was compared in eight patients with $\mathrm{AD}$ and six healthy controls. PET scanning detected more cerebral activation in patients with $\mathrm{AD}$, including increased activation of the basal ganglia that is known to be a central mediator of the itch-scratch cycle [42]. A similar study using histamine induction with eight $\mathrm{AD}$ patients and seven healthy controls utilized arterial spin labeling fMRI and found similar results in terms of significantly higher cerebral perfusion during acute itch among AD patients [43]. In chronic itch, brain imaging studies have shown that scratching induces overactivity in motor-related regions and the cerebral reward circuits. Furthermore, in two studies using arterial spin labeling fMRI to measure brain activity during active scratching, patients with chronic itch were found to have attenuated itch sensation in response to scratching, in addition to significant activation of cerebral structures involved in the central reward system [44, 45]. Taken together, these results demonstrate the pleasurable aspects of scratching to relieve both acute and chronic pruritus and suggest scratching can be addictive for patients with $\mathrm{AD}$.

It is important to note three key limitations of neurological imaging for acute and chronic pruritus associated with AD [41]. First, most of these studies represent small sample sizes and results vary on the basis of itch induction and imaging techniques. Second, no research has been published demonstrating differences in resting state between patients with $\mathrm{AD}$ and healthy controls. Instead, these techniques require an external itch stimulus (e.g., histamine-induced) to demonstrate significant differences that might not be exactly the same as in AD-induced itch. Third, methods like fMRI and PET take hours to perform and analyze and are thus inconvenient for patients and quite expensive to the health system. These considerations make brain imaging less practical for use in the clinical setting, especially for routine diagnostics or standard of care.

Despite these limitations, the findings collected from neurological imaging research could help guide future studies towards developing new treatments, including psychological interventions or non-invasive neurological procedures like transcranial direct current stimulation, which targets the pathways involved in the itch-scratch cycle and can have an antipruritic effect in $\mathrm{AD}$ [46]. An additional advantage of brain imaging is that it can be used to assess pruritus in AD patients while they are awake, unlikely many of the previously described methods that are limited to nocturnal measurements. For these reasons, it is critical that neurological imaging of pruritus is continued to better understand these pathways and 
identify potential targets to treat itch in $\mathrm{AD}$ $[47,48]$.

\section{CONCLUSIONS}

The variety of new and improved methods to evaluate pruritus in $\mathrm{AD}$ is welcomed by clinicians, researchers, and patients alike. While many of these technologies are promising, no single method discussed in this review can capture the full spectrum of the effects of itch on a patient's quality of life, ranging from the physical consequences like excoriation and lichenification to the mental impacts that can persist even in the absence of pruritus. It is therefore imperative to continue developing methods to quantify itch to accurately evaluate individual patients, guide treatment, and inform research on novel interventions to reduce the symptoms and burdens of itch. Future directions include next-generation smart devices as well as exploring new territories, such as identifying biomarkers that correlate to itch and machine-learning programs to identify itch processing in the brain. As these efforts continue, it will be essential to remain patientcentered by developing techniques that minimize discomfort, respect privacy, and provide accurate data that can be used to better manage itch in $\mathrm{AD}$.

\section{ACKNOWLEDGEMENTS}

Funding. No funding or sponsorship was received for this study or publication of this article.

Editorial Assistance. In addition, we would like to acknowledge Dr. Katrina Abuabara for her review of this manuscript.

Authorship. All named authors meet the International Committee of Medical Journal Editors (ICMJE) criteria for authorship for this article, take responsibility for the integrity of the work as a whole, and have given their approval for this version to be published.
Prior Presentation. No component of this manuscript has been posted, submitted, or published, or presented elsewhere. The table is original and has been produced by the authors for this particular publication.

Disclosures. Mary Patricia Smith, Karen Ly, Quinn Thibodeaux, and Thulasi Weerasinghe have nothing to disclose. Jashin $\mathrm{J}$. $\mathrm{Wu}$ is an investigator for AbbVie, Amgen, Eli Lilly, Janssen, Novartis; a consultant for AbbVie, Almirall, Amgen, Bristol-Myers Squibb, Celgene, Dermira, Dr. Reddy's Laboratories, Eli Lilly, Janssen, LEO Pharma, Novartis, Promius Pharma, Regeneron, Sun Pharmaceutical, and UCB, Valeant Pharmaceuticals North America LLC; and a speaker for AbbVie, Celgene, Novartis, Regeneron, Sanofi Genzyme, Sun Pharmaceutical, UCB, Valeant Pharmaceuticals North America LLC. Gil Yosipovitch conducted clinical trials or received honoraria for serving as a member of the Scientific Advisory Board and consultant of TREVI, Menlo, Sienna, Regeneron Sanofi, Galderma, Novartis, Pfizer, Kiniksa, AbbVie, and Eli Lilly and received research funds from Pfizer, Sun Pharma, Kiniksa, and Leo Pharma. Tina Bhutani has received research funding from the National Psoriasis Foundation and has served as a research investigator and/or consultant for Eli Lilly, Janssen, Merck, Celgene, and Regeneron. Wilson Liao is funded in part by grants from the National Institutes of Health (U01AI119125) and has served as a research investigator for Abbvie, Amgen, Janssen, Novartis, Pfizer, and Regeneron. Wilson Liao is a member of the journal's Editorial Board.

Compliance with Ethics Guidelines. This article is based on previously conducted studies and does not contain any studies with human participants or animals performed by any of the authors.

Data Availability. This review does not involve the generation or analysis of any original data sets, software codes, and/or models to make available for publication or in a publicly available repository. 
Open Access. This article is distributed under the terms of the Creative Commons Attribution-NonCommercial 4.0 International License (http://creativecommons.org/licenses/ by-nc/4.0/), which permits any noncommercial use, distribution, and reproduction in any medium, provided you give appropriate credit to the original author(s) and the source, provide a link to the Creative Commons license, and indicate if changes were made.

\section{REFERENCES}

1. Strom MA, Fishbein AB, Paller AS, Silverberg JI. Association between atopic dermatitis and attention deficit hyperactivity disorder in US children and adults. Br J Dermatol. 2016;175(5):920-9.

2. Schmitt J, Romanos M, Schmitt NM, Meurer M, Kirch W. Atopic eczema and attention-deficit/hyperactivity disorder in a population-based sample of children and adolescents. JAMA. 2009;301(7):724-6.

3. Thyssen JP, Hamann CR, Linneberg A, et al. Atopic dermatitis is associated with anxiety, depression, and suicidal ideation, but not with psychiatric hospitalization or suicide. Allergy. 2018;73(1):214-20.

4. Yaghmaie P, Koudelka CW, Simpson EL. Mental health comorbidity in patients with atopic dermatitis. J Allergy Clin Immunol. 2013;131(2):428-33.

5. Slattery MJ, Essex MJ, Paletz EM, et al. Depression, anxiety, and dermatologic quality of life in adolescents with atopic dermatitis. J Allergy Clin Immunol. 2011;128:668-71.

6. Silverberg JI. Practice gaps in pruritus. Dermatol Clin. 2016;34(3):257-61.

7. Yosipovitch G, Reaney M, Mastey V, et al. Peak pruritus numerical rating scale: psychometric validation and responder definition for assessing itch in moderate-to-severe atopic dermatitis. Br J Dermatol. 2019. https://doi.org/10.1111/bjd.17744.

8. Sanders KM, Nattkemper LA, Yosipovitch G. Advances in understanding itching and scratching: a new era of targeted treatments. F1000Res. 2016. https://doi.org/10.12688/f1000research.8659.1.

9. Potenzieri C, Undem BJ. Basic mechanisms of itch. Clin Exp Allergy. 2012;42(1):8-19.

10. Ikoma A, Rukwied R, Stander S, Steinhoff M, Miyachi Y, Schmelz M. Neuronal sensitization for histamine- induced itch in lesional skin of patients with atopic dermatitis. Arch Dermatol. 2003;139(11):1455-8.

11. Ikoma A, Steinhoff M, Stander S, Yosipovitch G, Schmelz M. The neurobiology of itch. Nat Rev Neurosci. 2006;7(7):535-47.

12. Paus R, Schmelz M, Biro T, Steinhoff M. Frontiers in pruritus research: scratching the brain for more effective itch therapy. J Clin Invest. 2006;116(5):1174-86.

13. Tivoli YA, Rubenstein RM. Pruritus: an updated look at an old problem. J Clin Aesthet Dermatol. 2009;2(7):30-6.

14. Stander S, Augustin M, Reich A, et al. Pruritus assessment in clinical trials: consensus recommendations from the International Forum for the Study of Itch (IFSI) Special Interest Group Scoring Itch in Clinical Trials. Acta Derm Venereol. 2013;93(5): 509-14.

15. Weisshaar E, Gieler U, Kupfer J, Furue M, Saeki H, Yosipovitch G. Questionnaires to assess chronic itch: a consensus paper of the special interest group of the International Forum on the Study of Itch. Acta Derm Venereol. 2012;92(5):493-6.

16. Moreau A, Anderer P, Ross M, Cerny A, Almazan $\mathrm{TH}$, Peterson B. Detection of nocturnal scratching movements in patients with atopic dermatitis using accelerometers and recurrent neural networks. IEEE J Biomed Health Inform. 2018;22(4):1011-8.

17. Price A, Cohen DE. Assessment of pruritus in patients with psoriasis and atopic dermatitis: subjective and objective tools. Dermatitis. 2014;25(6): 334-44.

18. Ebata T, Aizawa H, Kamide R. An infrared video camera system to observe nocturnal scratching in atopic dermatitis patients. J Dermatol. 1996;23(3): $153-5$.

19. Ebata T, Aizawa H, Kamide R, Niimura M. The characteristics of nocturnal scratching in adults with atopic dermatitis. Br J Dermatol. 1999; 141(1):82-6.

20. Park I, Lee K, Bishayee $\mathrm{K}$, Jeon HJ, Lee H, Lee U. Machine-learning based automatic and real-time detection of mouse scratching behaviors. Exp Neurobiol. 2019;28(1):54-61.

21. Umeda K, Noro Y, Murakami T, et al. A novel acoustic evaluation system of scratching in mouse dermatitis: rapid and specific detection of invisibly rapid scratch in an atopic dermatitis model mouse. Life Sci. 2006;79(22):2144-50.

22. Noro Y, Omoto Y, Umeda K, et al. Novel acoustic evaluation system for scratching behavior in 
itching dermatitis: rapid and accurate analysis for nocturnal scratching of atopic dermatitis patients. J Dermatol. 2014;41(3):233-8.

23. BusinessWire. LEO Science \& Tech Hub enters collaboration with Dina Katabi MIT, CSAIL to explore the use of radio signals for quantification of itch. https://www.businesswire.com/news/home/2018041 2005579/en/LEO-Science-Tech-Hub-Enters-Collabor ation-Dina. Accessed 1 Apr 2019.

24. Ebata T, Iwasaki S, Kamide R, Niimura M. Use of a wrist activity monitor for the measurement of nocturnal scratching in patients with atopic dermatitis. Br J Dermatol. 2001;144(2):305-9.

25. Bringhurst $\mathrm{C}$, Waterston $\mathrm{K}$, Schofield $\mathrm{O}$, Benjamin $\mathrm{K}$, Rees JL. Measurement of itch using actigraphy in pediatric and adult populations. J Am Acad Dermatol. 2004;51(6):893-8.

26. Benjamin K, Waterston K, Russell M, Schofield O, Diffey B, Rees JL. The development of an objective method for measuring scratch in children with atopic dermatitis suitable for clinical use. J Am Acad Dermatol. 2004;50(1):33-40.

27. Hon KL, Lam MC, Leung TF, et al. Nocturnal wrist movements are correlated with objective clinical scores and plasma chemokine levels in children with atopic dermatitis. Br J Dermatol. 2006;154(4): 629-35.

28. Feuerstein J, Austin D, Sack R, Hayes TL. Wrist actigraphy for scratch detection in the presence of confounding activities. Conf Proc IEEE Eng Med Biol Soc. 2011;2011:3652-5.

29. Wootton CI, Koller K, Lawton S, O'Leary C, Thomas KS. Are accelerometers a useful tool for measuring disease activity in children with eczema? Validity, responsiveness to change, and acceptability of use in a clinical trial setting. Br J Dermatol. 2012; 167(5):1131-7.

30. Petersen J, Austin D, Sack R, Hayes TL. Actigraphybased scratch detection using logistic regression. IEEE J Biomed Health Inform. 2013;17(2):277-83.

31. Lu TC, Fu CM, Ma MH, Fang CC, Turner AM. Healthcare applications of smart watches. A systematic review. Appl Clin Inform. 2016;7(3): 850-69.

32. Reeder B, David A. Health at hand: a systematic review of smart watch uses for health and wellness. J Biomed Inform. 2016;63:269-76.

33. Lee J, Cho D, Song S, Kim S, Im E, Kim J. Mobile system design for scratch recognition. 33rd Annual ACM Conference Extended Abstracts on Human Factors in Computing Systems. 2015. p. 1567-72.
34. Ikoma A, Ebata T, Chantalat L, et al. Measurement of nocturnal scratching in patients with pruritus using a smartwatch: initial clinical studies with the itch tracker app. Acta Derm Venereol. 2019; 99(3):268-73.

35. Iatklde $\mathrm{T}$, editor. Itch tracker: an application software turning wearable smart devices into a tool to measure nocturnal scratching. 9th World Congress on Itch 2017 Warsaw, Poland 15 Oct 2017.

36. Molenaar HA, Oosting J, Jones EA. Improved device for measuring scratching activity in patients with pruritus. Med Biol Eng Comput. 1998;36(2):220-4.

37. Stein H, Bijak M, Heerd E, et al. Pruritometer 1: portable measuring system for quantifying scratching as an objective measure of cholestatic pruritus. Biomed Tech (Berl). 1996;41(9):248-52.

38. Bijak M, Mayr W, Rafolt D, Tanew A, Unger E. Pruritometer 2: portable recording system for the quantification of scratching as objective criterion for the pruritus. Biomed Tech (Berl). 2001;46(5): 137-41.

39. Kogure T, Ebata T. Activity during sleep measured by a sheet-shaped body vibrometer and the severity of atopic dermatitis in adults: a comparison with wrist actigraphy. J Clin Sleep Med. 2018;14(2): 199-204.

40. Kogure T, Shirakawa S, Shimokawa M, Hosokawa Y. Automatic sleep/wake scoring from body motion in bed: validation of a newly developed sensor placed under a mattress. J Physiol Anthropol. 2011;30(3): 103-9.

41. Reich A, Szepietowski JC. Pruritus intensity assessment: challenge for clinicians. Expert Rev Dermatol. 2013;8(3):291-9.

42. Schneider G, Stander S, Burgmer M, Driesch G, Heuft G, Weckesser M. Significant differences in central imaging of histamine-induced itch between atopic dermatitis and healthy subjects. Eur J Pain. 2008;12(7):834-41.

43. Ishiuji Y, Coghill RC, Patel TS, Oshiro Y, Kraft RA, Yosipovitch G. Distinct patterns of brain activity evoked by histamine-induced itch reveal an association with itch intensity and disease severity in atopic dermatitis. Br J Dermatol. 2009;161(5): 1072-80.

44. Papoiu AD, Nattkemper LA, Sanders KM, et al. Brain's reward circuits mediate itch relief. A functional MRI study of active scratching. PLoS One. 2013;8(12):e82389.

45. Mochizuki H, Papoiu ADP, Nattkemper LA, et al. Scratching induces overactivity in motor-related 
regions and reward system in chronic itch patients. J Invest Dermatol. 2015;135(11):2814-23.

46. Mochizuki H, Schut C, Nattkemper LA, Yosipovitch G. Brain mechanism of itch in atopic dermatitis and its possible alteration through non-invasive treatments. Allergol Int. 2017;66(1):14-21.
47. Dong X. Peripheral and central mechanisms of itch. Neuron. 2018;98(3):482-94.

48. Tominaga M, Takamori K. Itch and nerve fibers with special reference to atopic dermatitis: therapeutic implications. J Dermatol. 2014;41(3):205-12. 